OBJETIVOS DE LAS PRÁCTICAS PROFESIONALES DE LA ESCUELA DE BIBLIOTECOLOGÍA $\quad \mathrm{Y}$ ARCHIVOLOGÍA

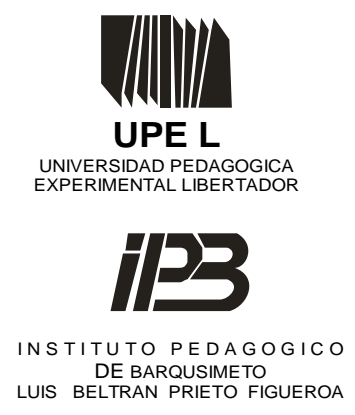

DE BARQUSIMETO
LUIS BELTRAN PRIETO FIGUEROA

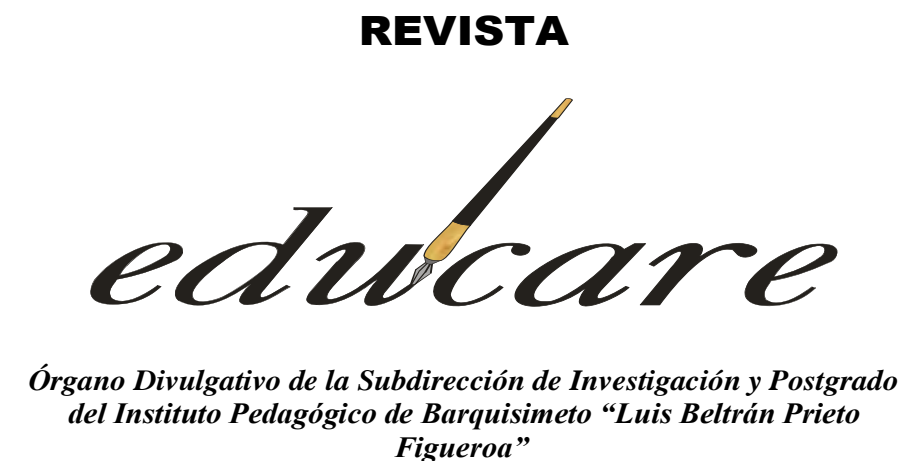

Figueroa"

\author{
BARQUISIMETO - EDO. LARA - VENEZUELA \\ NUEVA ETAPA \\ FORMATO ELECTRÒNICO \\ DEPOSITO LEGAL: ppi201002LA3674
}

ISSN: 2244-7296
Volumen 20 № 1

Enero - Abril 2016

\title{
OBJETIVOS DE LAS PRÁCTICAS PROFESIONALES DE LA ESCUELA DE BIBLIOTECOLOGÍA Y ARCHIVOLOGÍA DE LUZ
}

PROFESSIONAL PRACTICES OBJECTIVES OF THE BIBLIOTECHOLOGY AND ARCHIVOLOGY SCHOOL AT $\angle U Z$

Tania P. Vera, Roselin R. Esquivia, Yohaliseh C. Muñoz *

\author{
*UNIVERSIDAD DEL ZULIA (LUZ)
}


OBJETIVOS DE LAS PRÁCTICAS PROFESIONALES DE LA ESCUELA DE BIBLIOTECOLOGÍA $\quad$ Y ARCHIVOLOGÍA
Tania P. Vera, Roselin R. Esquivia, Yohaliseh C. Muñoz ( pp 29.-56)

\section{OBJETIVOS DE LAS PRÁCTICAS PROFESIONALES DE LA ESCUELA DE BIBLIOTECOLOGÍA Y ARCHIVOLOGÍA DE LUZ}

\section{PROFESSIONAL PRACTICES OBJECTIVES OF THE BIBLIOTECHOLOGY AND ARCHIVOLOGY SCHOOL AT LUZ}

\section{TRABAJO DE INVESTIGACIÒN}

Recibido:27-11-15

\author{
Tania P. Vera* \\ Roselin R. Esquivia** \\ Yohaliseh C. Muñoz \\ LUZ \\ Aceptado:11-02-16
}

RESUMEN

Este estudio es un avance de una investigación más amplia, y se enfoca en evaluar la correlación entre los objetivos de las prácticas profesionales II de la Licenciatura en Bibliotecología y Archivología de la Universidad del Zulia y el perfil de egreso, declarado en los dos últimos diseños curriculares (1995-2011 y 2012 hasta la actualidad). La metodología seguida fue el análisis de contenido de los objetivos de las PPII y del mencionado perfil, usando la propuesta de Abela (2000). La mayoría de los objetivos apuntan a la gestión técnica de documentos, soslayando el pensamiento crítico, uso de las TIC, ética, trabajo en equipo. Se recomienda ajustar los programas que orientan las PPII considerando como principal criterio las características del profesional que se aspira formar; y establecer mecanismos de monitoreo de los mismos, en aras de hacer las correcciones necesarias.

Palabras clave: Escuela de Bibliotecología y Archivología, perfil de egreso, objetivos de las Prácticas Profesionales de nivel

\begin{abstract}
This paper is an advance of a broader research, and it focuses on evaluating the correlation between the objectives of Professional Practices II of the Degree in Librarianship and Archivology of the University of Zulia and the output profile, declared in the last two Curricular designs (1995-2011 and 2012 to present). The content analysis of the objectives of the PPII and of the mentioned profile, using Abela's proposal (2000) was used. Most of the objectives point at technical management of documents, avoiding critical thinking, use of ICT, ethics and teamwork. It is recommended to adjust the programs that guide the PPII considering as the main criterion the characteristics of the professional that is aimed to form; and to establish mechanisms for monitoring them, in order to make the necessary corrections.
\end{abstract}

Keywords: School of Librarianship and Archivology, output profile, Level II Professional Practices objectives

\footnotetext{
*Tania Peña Vera. Licenciada en Bibliotecología y Archivología. Magister Scientiarium en Ciencias de la Comunicación, Doctora en Ciencias Humanas. Profesora titular de la Universidad del Zulia. tanipe7@gmail.com

**Roselin Rivas Esquivia. Licenciada en Bibliotecología y Archivología. Roselinrivas406@hotmail.com

***Yohaliseh Chávez Muñoz. Licenciada en Bibliotecología y Archivología. yohaliseh0912@gmail.com
} 
OBJETIVOS DE LAS PRÁCTICAS PROFESIONALES DE LA ESCUELA DE BIBLIOTECOLOGÍA $\quad$ Y ARCHIVOLOGÍA
Tania P. Vera, Roselin R. Esquivia, Yohaliseh C. Muñoz ( pp 29.-56)

\section{INTRODUCCIÓN}

El componente práctico de la formación profesional es importante por cuanto agrega elementos particulares que solamente desde la intervención de la realidad es posible adquirir, vale decir, las experiencias y vivencias obtenidas mediante una inserción controlada al campo laboral, permiten al estudiante profundizar en su propio aprendizaje, contrastar su conocimiento teórico con el empírico, descubrir sus capacidades y debilidades innatas y cognitivas, entender las dinámicas organizacionales y sociales propias de diferentes ambientes.

Son muchos los beneficios que se derivan de ese componente curricular, que es de carácter obligatorio para los institutos y colegios universitarios y las universidades de Venezuela.

Las modalidades que asumen las prácticas profesionales son diferentes, se les denomina: pasantías, seminarios, visitas estudiantiles, programas de gestión y cooperación tecnológica, mejoramiento de la educación técnica, formación de microempresarios, promoción de programas y convenios de asistencia técnica (Tovar, 2004). Lo importante de ello es que estén claros los objetivos que los estudiantes (pasantes) deben cumplir durante su ejecución, de manera que haya congruencia entre lo que se pretende, desde el punto de vista académico, y el beneficio que recibe el organismo que sirve de escenario laboral.

En tal sentido, se hace imperiosa la necesidad de declarar desde el propio currículo la articulación teoría - práctica que se quiere consolidar, para que ésta se traduzca en el apalancamiento del perfil profesional de un conjunto de sujetos en formación, que aspiran insertarse profesionalmente en el campo laboral, en aras de alcanzar su propio desarrollo personal y a la vez contribuir con la solución de problemas sociales, abordables desde su hacer como egresados universitarios.

Siendo el currículo un continuum, tal como lo señala Nolla (2001), y el proyecto educativo enfocado en la enseñanza y el aprendizaje, de naturaleza adaptable al desarrollo social, a las necesidades de los estudiantes y a los progresos científicos; que además define, estructura, organiza y orienta el sistema de objetivos, contenidos y formas que han de guiar la acción de profesores y estudiantes de un determinado nivel de enseñanza, en función de las demandas presentes en un contexto determinado; es preciso que establezca 
OBJETIVOS DE LAS PRÁCTICAS PROFESIONALES DE LA ESCUELA DE BIBLIOTECOLOGÍA Y ARCHIVOLOGÍA

Tania P. Vera, Roselin R. Esquivia, Yohaliseh C. Muñoz ( pp 29.-56)

puntualmente el alcance y peso que ha de tener el componente de las prácticas profesionales en el andamiaje de contenidos, porque resulta imprescindible para el delineamiento de un perfil profesional cónsono con la realidad social en la cual opera el currículo, y a cuyas necesidades responde a partir de la formación de talentos humanos.

En atención a esto, el diseño curricular de la Escuela de Bibliotecología y Archivología de la Universidad del Zulia (en adelante EBALUZ), de 1995.

Establece tres áreas de formación: la general, la profesional (básica-específica) y las prácticas profesionales comprendidas en tres niveles (I, II y III). Estas últimas constituyen un componente interdisciplinario en el cual se aplican y adquieren, en forma integrada los conocimientos, habilidades, destrezas, actitudes y valores correspondientes al perfil profesional del bibliotecólogo y archivólogo. (EBA-LUZ, 1995, pp. 28-29).

Los tres niveles de prácticas profesionales mencionados, se articulan de la siguiente manera:

- La práctica profesional I (PPI), ubicada en el primer semestre, inserta al estudiante en la realidad de su futura profesión, "vinculándolo con su campo de acción y proporcionándole a su vez una visión global de aspectos relacionados con el ejercicio de sus funciones como futuro gestor de la información” (Fernández, Battigelli, Luque, Gómez, Finol, Pérez, Pierre, Atencio, Gouviera, y Meléndez, 2002, pp. 1).

- $\quad$ La práctica profesional II (PPII) permite al estudiante apreciar los diferentes roles que debe cumplir como profesional de la información al vivenciar situaciones reales y concretas que conforman el quehacer profesional (Departamento Tecnología y Práctica Educacional, 1997). Las materias que integran este nivel son las siguientes:

○ La PPII: Automatización, ubicada en el 4to. semestre, con una duración de 4 horas semanales. En esta práctica se articulan los conocimientos adquiridos en Introducción al Procesamiento de Datos (1er. Semestre) e Informática General (2do. Semestre).

○ La PPII: Procesos Técnicos en Bibliotecas, ubicada en el 5to. semestre, con una duración semanal de 8 horas. Esta práctica articula los conocimientos teóricos de: Introducción a la Bibliotecología (1er. Semestre), Fuentes de Información 
OBJETIVOS DE LAS PRÁCTICAS PROFESIONALES DE LA ESCUELA DE $\begin{array}{llll}\text { BIBLIOTECOLOGÍA } & \mathrm{Y} & \text { ARCHIVOLOGÍA } & \text { DE }\end{array}$ Tania P. Vera, Roselin R. Esquivia, Yohaliseh C. Muñoz ( pp 29.-56)

(2do. Semestre), Formación de Colecciones (3er. Semestre) y la teoría de Procesos técnicos en bibliotecas (4to. Semestre).

○ La PPII Procesos Técnicos en Archivos, ubicada en el 6to. semestre, con una duración de 8 horas semanales. Esta práctica articula los conocimientos teóricos de: Introducción a la Archivología (1er. Semestre), Gestión documental (3er. Semestre) y la teoría de Procesos Técnicos en Archivos (5to. Semestre).

○ La PPII Análisis de la Información, ubicada en el 8vo. semestre, con una carga horaria semanal de 3 horas. Permite aplicar los conocimientos teóricos adquiridos en la materia Indización y Condensación (7mo. Semestre).

○ La PPII Seminario de Investigación, ubicada en el 8vo. semestre con una carga horaria semanal de 4 horas. Esta materia está dirigida al área investigativa mediante la cual el estudiante debe aplicar los conocimientos adquiridos en Metodología de la Investigación I (1er. Semestre) y II (2do. Semestre), Elaboración de proyectos (5to. Semestre) y Estadística (5to. Semestre), culmina con la ejecución de un trabajo de investigación sobre un tema relacionado con el área de las Ciencias de la Información.

- La práctica profesional III (PPIII) es un espacio de acción profesional que establece un acercamiento entre la teoría y el hacer práctico determinado por las particularidades del contexto donde éstas se ejecutan, haciendo posible que el estudiante integre sus conocimientos en experiencias reales que faciliten la aplicación de la teoría a la praxis. En la EBALUZ se establecen dos modalidades: una, es la pasantía propiamente dicha en la que el estudiante aplica habilidades y destrezas adquiridas a lo largo de la carrera, dentro de una organización que ha solicitado previamente pasantes para solucionar algún problema particular referido a la gestión de la información. La otra, es el trabajo de investigación, que consiste en una construcción teórica sobre algún aspecto de la realidad, siguiendo el método científico, bajo la tutoría de uno o varios profesores, su propósito es fomentar en los estudiantes el diseño de propuestas de soluciones creativas e innovadoras en alguna problemática particular relacionada con la especialidad, desde la investigación (Bracho y Peña, 2005). 
De estos niveles de prácticas, la presente investigación se centra en el nivel II, conformado por las cinco asignaturas mencionadas, de las cuales sólo las primeras cuatro son ejecutadas en centros de aplicación, es decir, en escenarios reales dentro o fuera del entorno universitario (EBALUZ, 1995); y son las que se tomaron como punto de partida para este estudio, en tanto que propician la oportunidad de interactuar tempranamente con el entorno laboral al que se integrarán los egresados en Bibliotecología y Archivología, y en ese sentido, de ellas se pueden derivar insumos curriculares valiosos para responder a las exigencias puntuales en materia del manejo de la información y perfilar así un egresado integral, eficiente y eficaz en su desempeño profesional. Lo que persigue cada una de las asignaturas que integran el nivel II de la práctica profesional se detalla a continuación:

- La PPII: Automatización, responde a necesidades informacionales, presentando una perspectiva sistemática constructivista, que involucra al estudiante como responsable de su propio aprendizaje (Portillo, 2003). Facilitando las herramientas necesarias para la ejecución de actividades que generen servicios y productos en el área de informática, por medio del uso de las tecnologías de información y comunicación para la búsqueda, selección y organización de la información obtenida.

- La PPII: Procesos Técnicos en Bibliotecas, intenta "desarrollar en el estudiante habilidades y competencias para el procesamiento, análisis y representación de la información y el conocimiento registrado en los diversos tipos de materiales bibliográficos y no bibliográficos", se fundamenta en una perspectiva holística (Bracho y Caldera, 2002, pp. 3).

- La PPII Procesos Técnicos en Archivos proporciona la oportunidad de aplicar los conocimientos adquiridos sobre el procesamiento del material documental que constituye el acervo de los archivos, con un enfoque sistemático, a través del cual se pretende obtener productos específicos derivados de la aplicación de teorías y técnicas referidas al procesamiento documental (Peña y Paredes, 2001, pp.s/p).

- La PPII Análisis de la Información, provee a los participantes la oportunidad de desarrollar actividades de carácter vivencial en escenarios y situaciones reales, integradoras de conocimientos, competencias y valores adquiridos en la asignatura 
OBJETIVOS DE LAS PRÁCTICAS PROFESIONALES DE LA ESCUELA DE BIBLIOTECOLOGÍA $\quad$ Y ARCHIVOLOGÍA
Tania P. Vera, Roselin R. Esquivia, Yohaliseh C. Muñoz ( pp 29.-56)

Indización y Condensación, aplicando metodologías, instrumentos, herramientas y productos del análisis documental al conocimiento registrado y producido en áreas específicas del saber. Se dirige al estudio de los procesos de construcción, significación, organización, representación y sociabilización de los saberes generados (Ferrer, 2010; Ferrer, s/f).

$\mathrm{Al}$ conocer estos propósitos surge la interrogante en cuanto al vínculo que debe existir entre los objetivos planteados en los programas de estas prácticas y el perfil profesional del bibliotecólogo y archivólogo declarado en el diseño curricular ¿Existe correspondencia entre ambos contenidos?

El componente de las prácticas profesionales o pasantías debe tener unos objetivos bien enfocados en relación a dos vertientes principales. Una de tipo académica, que se orienta a la consolidación del hacer profesional a partir de las experiencias controladas y propiciadas en el marco de la inserción de los estudiantes en entornos laborales reales; y que se espera que se traduzcan en un enriquecimiento vivencial, que impacte positivamente su dimensión cognitiva y actitudinal, y además contribuya a su mejor desempeño como futuros egresados universitarios.

Por otro lado, están los beneficios y soluciones que deben recibir los centros de aplicación (empresas e instituciones), desde las acciones ejecutadas por los pasantes insertados intencionalmente para resolver problemas puntuales, con la asistencia académica y guía de sus tutores, tanto académico como empresarial.

Los objetivos que han de guiar la ejecución de las prácticas necesitan ser, en principio, adecuadamente puntualizados por el ente académico conductor y planificador de la experiencia formativa de la pasantía y, a la vez, ser ampliamente conocidos por todos los actores intervinientes, vale decir, estudiantes, tutor académico y tutor empresarial e incluso por el personal de la organización que va a trabajar de cerca con el o los pasantes.

A partir de aquí se plantea como propósito de la investigación determinar la correlación entre los objetivos de aprendizaje contemplados en los programas de las PPII y el perfil de egreso de los estudiantes, declarado en el diseño curricular de la Escuela de Bibliotecología y Archivología de LUZ, la intención principal es detectar la afinidad entre esos elementos curriculares asociados muy puntualmente a la formación de los estudiantes 
OBJETIVOS DE LAS PRÁCTICAS PROFESIONALES DE LA ESCUELA DE BIBLIOTECOLOGÍA $\quad$ Y $\quad$ ARCHIVOLOGÍA
Tania P. Vera, Roselin R. Esquivia, Yohaliseh C. Muñoz ( pp 29.-56)

de las PPII. La existencia o no de correspondencia aportará datos interesantes para el análisis, además de ofrecer evidencias muy útiles para aplicar correctivos si fuese necesario.

Para la EBALUZ es de vital importancia establecer mecanismos de realimentación que permitan medir el alcance de los objetivos curriculares planteados, más allá de las notas obtenidas por los estudiantes en cada una de las prácticas, para verificar el logro de la configuración profesional que se espera en los cursantes de la mencionada licenciatura. Hasta el momento no ha habido un proceso de revisión o auditoría académica para constatar la vinculación existente entre los objetivos que orientan los programas del nivel II de prácticas profesionales y perfil profesional. Se espera con este estudio indagar objetivamente al respecto, usando como metodología el análisis del discurso, por cuanto se debe hacer una confrontación de los contenidos mencionados, en aras de medir su relación y congruencia.

\section{ARGUMENTACIÓN TEÓRICA}

En tanto que los egresados en Bibliotecología y Archivología de LUZ representan un erario para la sociedad moderna en lo que concierne a la gestión del conocimiento mediante el uso de tecnologías de información y comunicación, es imprescindible vigilar la consolidación de su perfil profesional, manifiesto en el desarrollo de las competencias, habilidades, destrezas, aptitudes y actitudes necesarias para erigirse como un agente de mediación informativa y cognitiva.

El aporte de las teorías a la solución del problema expuesto se enfoca en esclarecer los factores que intervienen en él y su concepción científica. Dentro de las bases teóricas se contemplan las posturas de diferentes autores sobre aspectos como el currículo, su evaluación y diseño, los objetivos de aprendizaje, el perfil de egreso, y sus dimensiones, las prácticas profesionales, sus fases, dimensiones, evaluación, valoración y ventajas.

Algunos de los teóricos tomados para sustentar lo que es el currículo, su evaluación, tipos y diseños fueron: Inciarte (2005), Tapia (2003), Florez (2002), Posner (2004), Casarini (2001), López (2011), Navarro (s.a), Berger y Kam (1996). En general, se define al currículo como intermediario entre la teoría y el contexto, vale decir, la realidad; por lo 
OBJETIVOS DE LAS PRÁCTICAS PROFESIONALES DE LA ESCUELA DE BIBLIOTECOLOGÍA $\quad$ Y ARCHIVOLOGÍA
Tania P. Vera, Roselin R. Esquivia, Yohaliseh C. Muñoz ( pp 29.-56)

que está expuesto a cambios sociales, y es en esencia adaptativo, evolutivo y variante. Su tipología de evaluación van aparejadas entre: lo formal y el perfil de egreso; lo real y las estrategias docentes; lo oculto y los valores y actitudes. El diseño curricular es elaborado en virtud de la razón de ser del currículo. Representa ideas, acciones, objetivos que guían la ejecución del plan de estudio. Éste muestra al docente cómo deberá ejecutar los programas de las asignaturas en el proceso formativo.

Para fundamentar los objetivos de aprendizaje se tomó a Zarzar (1994) y Morales (s.a), quienes los asumen como las líneas generales que van a orientar el trabajo del docente según cada programa de asignatura, y son de tipo informativo y formativo que en conjunto incluyen las etapas del proceso educativo desde que el estudiante adquiere conocimientos, métodos, habilidades, destrezas, actitudes y valores, hasta la aplicación teórico-práctica contemplada en el perfil de egreso.

En cuanto al perfil de egreso y sus dimensiones se emplearon los postulados de: Casarini (2001), Vílchez (1991), Torres (s.a), Coon (2005), Hawes y Corvalán (2005), Canquíz e Inciarte (2007) y Navarro (s.a). Sus planteamientos señalan al perfil de egreso como unificador de elementos prácticos y teóricos que describen el desempeño de un egresado en términos de habilidades cognitivas, valóricas y actitudinales pertinentes, y que le permiten responder ante las exigencias de la sociedad desenvolviéndose en cualquier escenario previsto. Dentro de sus componentes destacan las prácticas profesionales, porque a través de ellas se viabiliza la inserción de los estudiantes en escenarios reales, y promueve la aplicación de sus conocimientos a situaciones laborales propias de su área de acción profesional.

Vidal (2009) expresa que los perfiles profesionales "evolucionan y cambian según la demanda ocupacional y el mercado de trabajo, por tanto son dinámicos” (pp. 5), ya que se modifican constantemente debido a factores de contexto, socioculturales, psicológicos y pedagógicos. En tal sentido, es imprescindible la evaluación constante de los elementos inherentes a la formación académica y profesional de los estudiantes, la cual requiere, entre otras cosas, el monitoreo de los objetivos que persigue cada asignatura, incluidas las prácticas profesionales, para detectar debilidades y fortalezas que se puedan corregir o potencializar, según sea el caso. 
OBJETIVOS DE LAS PRÁCTICAS PROFESIONALES DE LA ESCUELA DE BIBLIOTECOLOGÍA $\quad$ Y $\quad$ ARCHIVOLOGÍA
Tania P. Vera, Roselin R. Esquivia, Yohaliseh C. Muñoz ( pp 29.-56)

"Existe hoy en día una tendencia de fijar estándares nacionales para los egresados de las diferentes carreras, con el fin de constituir un marco referencial de aquellas competencias que debieran tener", (Vidal, 2009, pp.5). En atención a esto, las entidades educativas definen perfiles profesionales de egreso para guiar la consolidación de competencias, actitudes y la adquisición de conocimientos necesarios en la formación de sus estudiantes; en esto juega un papel importante el complemento práctico.

En los diseños curriculares se particularizan las competencias específicas que se aspira desarrollar en los estudiantes antes de graduarse, para ello se implementan recursos educativos, que permitan alcanzar el perfil deseado, uno es la inclusión de prácticas profesionales que buscan servir como elementos catalizadores e integradores de la formación académica, e incluso ir más allá, interviniendo también en la dimensión humana y actitudinal de los estudiantes.

Las prácticas profesionales constituyen los espacios curriculares idóneos en los que ocurre la integración de conocimientos, y delinean en gran manera el perfil de egreso de los estudiantes. Por ende, los objetivos de aprendizaje orientadores de su ejecución deben partir de esa concepción profesional que se aspira formar.

Los objetivos contenidos en los programas que realiza cada docente para las asignaturas que dicta, incluidas las materias prácticas, deben estar en total congruencia con el perfil profesional delineado, y así desde cada uno de aquellas se hagan aportes progresivos en la formación académica del individuo; dejando en claro que el alcance del perfil de egreso, depende únicamente del esfuerzo y compromiso de cada estudiante.

Lo concerniente a las prácticas profesionales, los modelos existentes, sus fases, dimensiones organizativas, evaluación y valoración se sustentó en las perspectivas de autores como: Castellano (2008), Universidad Central de Venezuela (s.a), Perrenoud (2004), Zabalza (2013), Santibáñez y Montero (1998); así como la Ley Orgánica de Educación (2009), Ajá y Albaladejo (2000), Lau (2005), Bligh (2000), Kaplan (2004). En resumen, se asume a la práctica profesional como un espacio académico de observación, participación y actuación en situaciones reales que fomentan en el estudiante la madurez, confianza, preparación profesional necesaria. Sus fases deben constituir una secuencia lógica de ejecución de acciones, que van desde saber que hará el estudiante, dónde será 
asignado, qué realizará, cómo lo llevará a cabo, con quién deberá contar y hasta cuándo estará desarrollando las prácticas.

Para complementar lo expuesto por los autores se agregan las fases de las prácticas profesionales que plantea Castellano (2008) a saber:

-Planificación: fines, objetivos, recursos, espacios, condiciones (personales, materiales, organizativos y de intervención).

-Programación: guías con distribución de funciones, entre la institución receptora y los practicantes, garantizando el doble apoyo: asesores expertos y profesores tutores.

-Realización de actividades: análisis, métodos, instrumentos, seguimientos (evaluación colegiada de la práctica profesional)

-Recuperación: control y evaluación (instrumentos, retroalimentación e informes).

La revisión de este autor coincide con la de los mencionados anteriormente, en cuanto al entrelazamiento de una fase con otra en una secuencia progresiva de ejecución de acciones.

En lo que respecta a las dimensiones de las prácticas profesionales, se conciben como integradoras de las modalidades, las fases, las etapas de evaluación, los participantes (estudiantes, profesores y representantes de las instituciones o empresas), de la visión del diseño curricular, del plan de estudio, de los programas, sus objetivos y su evaluación.

El proceso de evaluación de las prácticas profesionales tiene como finalidad determinar en qué medida se ha logrado lo previsto, posibilitando la determinación de las desviaciones y la adopción de acciones correctivas que garanticen el cumplimiento adecuado de los objetivos. La valoración es parte del proceso evaluativo, y considera progresivas aproximaciones al sujeto evaluado.

El aporte de las teorías abordadas es el esclarecimiento conceptual de los factores que intervienen en el problema planteado, pues, han permitido caracterizarlos y entender su vinculación en el entramado de esta investigación en particular. 
OBJETIVOS DE LAS PRÁCTICAS PROFESIONALES DE LA ESCUELA DE BIBLIOTECOLOGÍA $\quad$ Y ARCHIVOLOGÍA
Tania P. Vera, Roselin R. Esquivia, Yohaliseh C. Muñoz ( pp 29.-56)

\section{ASPECTOS METODOLÓGICOS}

La metódica planteada está basada en el análisis de contenido, pues este estudio parte de la necesidad de cotejar los objetivos de aprendizaje planteados en los programas de las prácticas profesionales de nivel II (PPII) de la EBALUZ, y el perfil de egreso declarado en el diseño curricular de la licenciatura en Bibliotecología y Archivología que administra la Universidad del Zulia. En tal sentido, el desarrollo de la investigación supone la ejecución del método de análisis, que consiste según Rodríguez (s/a, pp. s.f) en descomponer algo que se desea conocer a profundidad en cada una de sus partes esenciales para examinarlas en forma individual.

Se supone, que los objetivos definidos en los programas de cada PPII contribuyen a delinear el perfil que se pretende para los egresados de la mencionada carrera, es decir, que van a la par con lo que se decreta en cuanto a la excelencia formativa que se aspira del futuro profesional. En tal sentido, el contenido semántico de las palabras empleadas en la redacción de los objetivos y del perfil juega un papel fundamental. Por ello se recurre al análisis de texto o contenido, que radica en el estudio explícito de lo que se expresa concretamente, tanto en los objetivos de aprendizaje de los programas de PPII como en el perfil de egreso, para identificar los rasgos distintivos, incongruencias, disimilitudes, correlaciones, reciprocidad o conexión existente entre los parámetros determinados.

En cuanto a la población y muestra, que es el universo sobre el cual se pretende generalizar resultados en función de sus características específicas (Chávez, 2007). La mismas están conformadas por los programas de estudios de vigentes del nivel II de las prácticas profesionales ofrecidos por la EBALUZ en el momento de realizarse el estudio, vale decir, PPII: Automatización, PPII: Procesos Técnicos en Bibliotecas, PPII: Procesos Técnicos en Archivos y PPII: Análisis de la Información. En atención al limitado número de entes a estudiar, no se hará el cálculo de una muestra.

Detallando un poco lo referente a la técnica e instrumentos de recolección de datos a emplear en esta investigación, Krippendorff (1990, pp. 28) define al análisis de contenido como "una técnica de investigación destinada a formular, a partir de ciertos datos, inferencias reproducibles y válidas que puedan aplicarse a su contexto". Se trata de estudiar 
OBJETIVOS DE LAS PRÁCTICAS PROFESIONALES DE LA ESCUELA DE BIBLIOTECOLOGÍA $\quad$ Y ARCHIVOLOGÍA
Tania P. Vera, Roselin R. Esquivia, Yohaliseh C. Muñoz ( pp 29.-56)

los elementos seleccionados en su dimensión discursiva y generar supuestos que viabilicen la comprensión profunda del contenido de las unidades de análisis elegidas para tales fines.

Por otra parte, Berelson (1952, pp. resumen) sostiene que el análisis de contenido es "una técnica de investigación para la descripción objetiva, sistemática y cuantitativa del contenido manifiesto de la comunicación”. Según este autor el análisis de contenido se debe someter a reglas como la "objetividad", que es el empleo de medios que puedan ser utilizados por otros investigadores de manera que los resultados sean idóneos para ser verificados; y a la "sistematización" que se refiere a las pautas ordenadas que abarquen el total del contenido observado.

El análisis de contenido es entonces, un procedimiento que permite descomponer textos en sus elementos semánticos más esenciales y esto posibilita la identificación de vínculos o disociaciones entre ellos.

\section{Pasos para realizar el análisis de contenido}

Sierra (1995, pp. s.p), propone los siguientes pasos para realizar un estudio de contenido: a.- "el muestreo (de las fuentes documentales a ser analizadas); b.- determinación de unidades de análisis; c.- elección de categorías; d.- confección del cuadro de recogidas de datos".

Por su parte, Mayintz y col. (1980, pp. s.p), señalan que hay seis fases en el análisis de contenido: a.- "preparación teórica; b.- determinación de la relevancia de un texto; c.determinación de las unidades lingüísticas; d.- desarrollo del esquema de categorías de análisis; e.- recuento, formación de índices y comprobación de hipótesis; f.- fiabilidad y validez".

Por otro lado, Abela (2000, pp. s/p), menciona la existencia de cinco componentes del análisis de contenido. Indicando que todo proyecto o plan de investigación en el que se emplee la técnica de análisis de contenido ha de distinguir varios pasos diferentes en su proceso, como lo son: a. "Determinar el objeto o tema de análisis; b. Determinar las reglas de codificación; c. Determinar el sistema de categorías; d. Comprobar la fiabilidad del sistema de codificación-categorización; e. Inferencias." 
OBJETIVOS DE LAS PRÁCTICAS PROFESIONALES DE LA ESCUELA DE BIBLIOTECOLOGÍA $\quad$ Y $\quad$ ARCHIVOLOGÍA
Tania P. Vera, Roselin R. Esquivia, Yohaliseh C. Muñoz ( pp 29.-56)

Al mirar en paralelo las propuestas de Sierra (1995), Mayintz y col. (1980) y Abela (2000), se puede precisar que se complementan entre sí. Y su integración ofrece un método más amplio y complejo para investigar, estudiar y analizar de manera objetiva, sistemática y profunda cualquier contenido. Sin embargo, se tomó como base la de Abela (2000), ya que explica detalladamente cada paso que a seguir y es mucho más fácil de comprender. A pesar de no contemplar la fase previa de forma explícita, incluye al inicio una serie de preguntas cuyas respuestas permiten contextualizar el tema de análisis para adentrarse luego al desglose propiamente dicho. El planteamiento de este autor puede asumirse bajo un esquema cualitativo o cuantitativo como de producción de datos o de análisis o como investigación descriptiva o explicativa.

Como recomendación general Bartolomé, (1981, pp. 251), indica que debe evitarse caer, durante el análisis de contenido, en tres fuentes de error importantes, a saber: extracción de la palabra de su contexto, arbitrariedad subjetiva en la categorización, y de otorgar primacía a lo cuantitativo sobre lo cualitativo en la interpretación de los resultados. Todo ello entorpece la interpretación adecuada de los núcleos de significado expuestos en el tex to objeto de estudio.

\section{Despliegue del análisis de contenido}

Abela (2000) establece que para iniciar el análisis se formulen ciertas preguntas, tales como:

“... ¿Qué se quiere investigar?, ¿qué bibliografía o conocimientos previos existen?, ¿en qué teoría o marco teórico encaja?, ¿qué texto o textos se van a utilizar?, y ¿cuál es la unidad de análisis que se va a utilizar?” (pp. 11)

- A la pregunta ¿qué se quiere investigar? Se responde que es la correlación de los objetivos contemplados en los programas de las prácticas profesionales II y el perfil de egreso declarado en el diseño curricular de la licenciatura en Bibliotecología y Archivología que administra la Universidad del Zulia.

- En cuanto a la bibliografía o conocimientos previos existentes y en qué teoría encaja, se responde que en el marco teórico que sustenta esta investigación, se desglosaron aspectos referidos al diseño curricular, los objetivos de aprendizaje, 
y el perfil de egreso de los estudiantes, usando los argumentos de autores como Flórez (2002, pp.12), Casarini (2001, pp. 96), Canquiz e Inciarte (2007, pp. 18), Zarza (1994, pp. 5) y Morales (s/a).

- Como planteamientos adyacentes referidos a la correlación entre ambos elementos, se toman los aportes teóricos de Vidal (s/a) y Cruz (2001), que abordan la relación entre el quehacer docente y el perfil de egreso basado en competencias y entre los hábitos de estudio, actitudes, autoestima y el rendimiento académico de estudiantes de enfermería. Siguiendo un procedimiento de relación de investigación, aun y cuando no se contemple la misma metodología; no obstante, permite acercarse al fenómeno en estudio.

- Para responder la pregunta ¿Qué textos se van a utilizar? se revela que son los siguientes:

o Programa de PPII: Automatización, elaborado por Portillo (2003).

○ Programa de PPII: Procesos técnicos en archivos, elaborado por Peña y Paredes (2001).

○ Programa de PPII: Análisis de la información, elaborado por Ferrer (2010).

○ Programa de PPII: Procesos técnicos en bibliotecas, elaborado por Bracho y Caldera (2002).

○ Sinópticos de los nuevos programas de PPII del diseño del 2012.

- Diseño Curricular Escuela de Bibliotecología y Archivología de la Universidad del Zulia 1995 vigente hasta el 2011 y el que entró en vigencia en el año 2012 y los programas sinópticos de: PPII Teleinformática, PPII Análisis de la información I, PPII Análisis de la información II y PPII Análisis de la información III.

- En cuanto a las unidades de análisis, las mismas están constituidas por los objetivos de los programas de prácticas profesionales II y el perfil de egreso declarado en el diseño curricular desde 1995 hasta 2011, y el que entró en vigencia a partir del 2012. Los mencionados elementos forman una unidad de registro, por cuanto son segmentos específicos. 
OBJETIVOS DE LAS PRÁCTICAS PROFESIONALES DE LA ESCUELA DE BIBLIOTECOLOGÍA $\quad$ Y ARCHIVOLOGÍA
Tania P. Vera, Roselin R. Esquivia, Yohaliseh C. Muñoz ( pp 29.-56)

Como siguiente fase Abela (2000), propone el uso de un sistema de codificación que él mismo configura, y que se tomó casi en su totalidad para esta investigación. A continuación se desglosa.

\section{Sistema de codificación}

$\checkmark \quad \mathrm{P}=$ Presencia ó NP= No Presencia

Según el autor en cuestión la presencia o ausencia de los elementos de un texto pueden ser importante o significativo en aras de conocer inclinaciones explicitas o subyacentes.

$\checkmark \quad F=$ Frecuencia (número de apariciones): la frecuencia es la medida más utilizada generalmente, válida en unos casos y en otros no. La importancia de una unidad de registro crece con su frecuencia de aparición.

$\checkmark \quad \mathrm{FP}=$ Frecuencia ponderada: se refiere al otorgamiento de mayor importancia de unos elementos sobre otros; se representará de la siguiente forma (Ax2) alta y (Bx1) baja importancia. Cuando se supone que la aparición de uno o varios elementos tiene más importancia que los demás, se suele recurrir a sistemas de ponderación.

$\checkmark \quad$ I= Intensidad: según el planteamiento de Osgood (1980) este aspecto es posible medirlo a través del tiempo verbal utilizado de la siguiente manera: tiempo verbal condiciona, indica menos intensidad; tiempo verbal futuro indica mediana intensidad; tiempo verbal imperativo indica más intensidad. Este código tampoco será utilizado porque en la redacción de objetivos de aprendizaje se sigue una línea discursiva coherente con el progreso o avance académico de los estudiantes, por ende, no existen variedad en las formas enunciativas empleadas que pudieran arrojar datos de interés para este estudio.

$\checkmark$ DI= Dirección, esta puede ser + positiva ó + negativa, para determinar este aspecto es necesario establecer un sistema de codificación donde se vea reflejado el sentido bidireccional del texto. Este código no se utiliza, ya que la dirección establece que las categorías deben indicarse como negativas o positivas, según sea el caso, y en este, todas son designadas como positivas ya que contribuyen a la formación académica y profesional del estudiante, y además su ejecución representa un aporte a las unidades de información y a la sociedad en general. 
OBJETIVOS DE LAS PRÁCTICAS PROFESIONALES DE LA ESCUELA DE

$\begin{array}{lcc}\text { BIBLIOTECOLOGÍA } & \text { Y ARCHIVOLOGÍA } \\ \text { Tania P. Vera, Roselin R. Esquivia, Yohaliseh C. Muñoz ( pp 29.-56) }\end{array}$

$\checkmark \mathrm{CO}=$ Contingencia: es la presencia simultánea en un momento dado de dos o más unidades de registro en diferentes niveles de códigos o de contextos. Esta se evaluará entre los objetivos declarados en los cuatro programas de PPII correspondientes al diseño curricular 1995 y luego entre los objetivos expresados en los sinópticos de los nuevos programas de PPII del diseño del 2012.

\section{Sistema de categorías}

En esta investigación las categorías se toman de perfil profesional o perfil de egreso, en tanto que este constructo dicta la pauta para el análisis de contenido de los objetivos de las PPII, por declarar los roles, las competencias, los rasgos actitudinales, cognitivos, de habilidades y profesionales que se esperan formar en los estudiantes de la Licenciatura en Bibliotecología y Archivología de LUZ. A continuación se presenta el sistema de categorías correspondiente al diseño curricular 1995 y luego el de 2012.

\section{Sistema de categorías del diseño curricular de $\mathbf{1 9 9 5}$}

1. Roles específicos:

\section{A. Técnico}

a- Gestión técnica de documentos (recopilación, adquisición, ordenación, selección y clasificación de documentos) aplicar métodos, técnicas y procedimientos, diseñar políticas y procedimientos para la gestión técnica).

b- Gestión tecnológica de la información (aplicación de TIC)

B. Analista

a. Gestión de contenidos (análisis y síntesis de contenidos, generación de productos informacionales)

b. Orientación y apoyo a usuarios

c. Localización y administración de fuentes de información

\section{Gerente}

a. Gerencia de unidades de información (aplicación de principios administrativos, establecimiento de políticas, métodos y procedimientos, gestión de recursos humanos, económicos, de infraestructura y tecnológicos) 
b. Mercadeo de productos y servicios de información

D. Investigador

a. Desarrollo de procesos investigativos

b. Aplicación de los resultados del proceso investigativo en la resolución de problemas existentes en unidades y centros de información

c. Apoyo a la investigación a través de las TIC

E. Promotor social

a. Gestión cultural

b. Actividades de integración comunal a los servicios de información

c. Alfabetización informacional y tecnológica

2. Conocimientos sobre:

A. Gestión de información

B. Funcionamiento de servicios de información

C. Impacto de la información en el sector socioproductivo

D. Gestión de recursos humanos

E. Liderazgo

3. Habilidades para:
A. Aplicar procedimientos administrativos/gerenciales
B. Aplicar instrumentos de investigación
C. Manejar TIC
D. Establecer relaciones con el entorno

4. Actitudes positivas hacia:
A. Los cambios sociales y tecnológicos
B. Acciones de calidad
C. Solución de problemas
D. Trabajo en equipo
E. Desempeño ético

\section{Sistema de categorías del diseño curricular de 2012}

1. Competencias generales: 
A. Investigación

a. Desarrolla procesos de investigación para el manejo de hechos, ideas, significados y fenómenos con una actitud transformadora, crítica y reflexiva

B. Tecnologías de información y comunicación

a. Utiliza las tecnologías de la información y la comunicación con valores éticos, aprovechando las ventajas que ofrece cada una según el contexto de uso, respondiendo a las tendencias mundiales de desarrollo tecnológico, científico y cultural.

C. Responsabilidad social y participación ciudadana

a. Participa activa y solidariamente en el diseño y ejecución de proyectos pertinentes para el desarrollo de la comunidad con responsabilidad social.

D. Pensamiento crítico

a. Asume una actitud crítica en la toma de decisiones para la detección y resolución de problemas, aceptando estándares consensuados socialmente con independencia de criterios.

E. Comunicación

a. Intercambia información con sus interlocutores, utilizando correcta y adecuadamente el lenguaje y los diversos medios, formas, procedimientos e instrumentos de la comunicación.

F. Ecología y ambiente

a. Responde a una racionalidad ambiental aplicando la normativa nacional e internacional que rige la materia, en cuanto a los procesos bióticos y abióticos que pueden afectar el medio ambiente, a fin de hacer un uso racional de los recursos en su ámbito personal, profesional a favor del colectivo.

G. Ética

a. Actúa en todos los ámbitos de la vida consecuentemente con los valores morales y las buenas costumbres, asumiendo con responsabilidad las consecuencias de sus propias acciones. 
OBJETIVOS DE LAS PRÁCTICAS PROFESIONALES DE LA ESCUELA DE BIBLIOTECOLOGÍA $\quad$ Y $\quad$ ARCHIVOLOGÍA
Tania P. Vera, Roselin R. Esquivia, Yohaliseh C. Muñoz ( pp 29.-56)

2. Competencias específicas:

\section{A. Cognitivas}

a. Analiza los modelos teóricos para la organización y representación de la información y el conocimiento

b. Identifica las tendencias sobre organización y representación de la información y el conocimiento.

c. Distingue los diferentes niveles de procesamiento de la información

d. Analiza modelos y procesos gerenciales aplicables en los servicios y unidades de información.

e. Distingue los diversos tipos de servicios y productos de información.

f. Identifica los componentes de la gestión integral de los servicios para satisfacer necesidades formativas e informativas de los usuarios

g. Comprende el proceso histórico de las instituciones y soportes de información como patrimonio de la humanidad.

h. Conceptualiza el patrimonio documental desde la perspectiva de los bienes culturales tangibles que constituyen la memoria colectiva.

i. Identifica los principios teórico-metodológicos y técnicos de la conservación preventiva del patrimonio documental y su difusión.

j. Relaciona la acción de las organizaciones de conocimiento (bibliotecas, archivos, centros de documentación y museos) para afianzar valores de identidad nacional.

B. Procedimentales

a. Ejecuta la organización y representación de la información y el conocimiento en distintos soportes y en diversos tipos de unidades, sistemas y servicios de información, considerando estándares.

b. Diseña herramientas para la organización y representación de la información y el conocimiento.

c. Aplica diferentes técnicas y procedimientos para la organización y representaciones de la información y el conocimiento, sustentadas en las Tecnologías de la Información. 
d. Diseña planes, políticas y estrategias para impulsar el desarrollo y proyección del servicio que dirige con el propósito de responder a las demandas del sector donde se inserta.

e. Establece alianzas estratégicas con diversos organismos públicos y privados y con otros servicios de información.

f. Gestiona el talento humano de las unidades y servicios de información Ejecuta métodos, procedimientos y técnicas de conservación preventiva del patrimonio documental existente en la unidad a su cargo, considerando criterios y estándares.

g. Estima el valor económico de documentos impresos y digitales.

h. Diseña planes para la difusión y apropiación del patrimonio documental

\section{Actitudinales}

a. Valora su importancia como analista de información para promover la socialización del conocimiento.

b. Asume una posición crítica en relación con la importancia de la organización y representación de la información y el conocimiento para la maximización de su acceso.

c. Valora la dimensión ética y de responsabilidad social de la gestión integral de las unidades y servicios de información.

d. Reconoce la importancia de su rol como gerente social que promueve acciones relacionadas con el desarrollo humano integral e incluyente.

e. Promueve campañas de concienciación acerca de la importancia de la conservación del patrimonio documental como vía para el afianzamiento de los valores de identidad nacional.

f. Asume el patrimonio documental de la unidad que dirige como parte de la memoria colectiva mediante la cual se afianza la identidad nacional.

g. Valora la importancia de la función de promoción socio-cultural de las unidades de información 
OBJETIVOS DE LAS PRÁCTICAS PROFESIONALES DE LA ESCUELA DE $\begin{array}{lllll}\text { BIBLIOTECOLOGÍA } & \text { Y } & \text { ARCHIVOLOGÍA } & \text { DE }\end{array}$ Tania P. Vera, Roselin R. Esquivia, Yohaliseh C. Muñoz ( pp 29.-56)

Los números y letras utilizados para enumerar las categorías se emplearon para identificarlas de manera individual durante el análisis de contenido.

\section{Fiabilidad del sistema de codificación- categorización}

De acuerdo con Abela (2000),

la importancia de la fiabilidad procede de la seguridad de ofrecer que los datos han sido obtenidos con independencia del suceso, instrumento o persona que los mide. Por definición, los datos fiables son aquellos que permanecen constantes en todas las variaciones del proceso analítico. (pp.15)

Los datos sobre fiabilidad exigen que dos codificadores, como mínimo, según indica Kripperdorf (1990), describan de forma independiente un conjunto posiblemente amplio de unidades de registro en los términos de un lenguaje común. La fiabilidad se expresa como una función del acuerdo alcanzado entre los codificadores sobre la asignación de las unidades a las diversas categorías. Si dicho acuerdo es total para todas las unidades, está garantizada la fiabilidad; por el contrario, si es mayor que el correspondiente al azar, la fiabilidad es nula.

El sistema de categorías expuesto se elaboró preliminarmente tomando solo lo expresado en los diseño curriculares 1995 y 2012, posteriormente fue sometido a la consulta de los profesores expertos en las distintas áreas curriculares. De esta revisión se hicieron algunos ajustes que permitieron condensar en descriptores mutuamente excluyentes las categorías inicialmente propuestas. Luego de ello, el sistema fue usado a manera de ensayo por los miembros del equipo de investigación demostrando ser coherente y de fácil manejo por cada uno de ellos. Con esto se cubrió la fase de fiabilidad sugerida por el autor en cuestión.

Una vez construidos los sistemas de categorías correspondientes a cada diseño curricular, se procedió a determinar la correlación de los objetivos de las PPII con el perfil de egreso, cotejando la afinidad existente entre ellos.

\section{Hallazgos/resultados}

De la correlación entre el diseño curricular y los programas de las PPII vigentes hasta 
OBJETIVOS DE LAS PRÁCTICAS PROFESIONALES DE LA ESCUELA DE $\begin{array}{lllll}\text { BIBLIOTECOLOGÍA } & Y & \text { ARCHIVOLOGÍA } & \text { DE } & \text { LUZ }\end{array}$ Tania P. Vera, Roselin R. Esquivia, Yohaliseh C. Muñoz ( pp 29.-56)

el 2011, se obtuvieron los siguientes resultados; con respecto a la frecuencia (F) de aparición de las categorías en los objetivos de los cuatro programas analizados:

1. 2A Conocimiento sobre-gestión de información (12)

2. 1 Aa Rol técnico-gestión técnica de documentos (9)

4B Actitudes positivas hacia acciones de calidad (9)

3. 3D Habilidades para establecer relaciones con el entorno (8)

4. 1Dc Rol investigador-apoyo a la investigación a través de las TIC (7)

5. 1Ba Rol analista-gestión de contenido (6)

1Bc Rol analista-localización y administración de fuentes de información (6)

4C Actitudes positivas hacia la solución de problemas (6)

Los datos obtenidos revelan que el conocimiento que más se promueve es el referido a la gestión de información, así como el desempeño del rol técnico-gestión técnica de documentos y las actitudes positivas hacia acciones de calidad que debe adquirir el estudiante.

Las categorías con presencia simultánea en los objetivos de la mayoría de los programas analizados son:

1Aa: Rol técnico-gestión técnica de documentos

1Bc: Rol analista: localización y administración de fuentes de información

1Dc: Rol investigador: apoyo a la investigación a través de las TIC

2A: Conocimiento sobre-gestión de información

3D: Habilidades para establecer relaciones con el entorno

4B: Actitudes positivas hacia acciones de calidad

4C: Actitudes positivas hacia la solución de problemas

\section{Derivación de inferencias del primer grupo de documentos}

Según Abela (2000, pp.19), inferir es explicar, deducir lo que hay en un texto. El analista de contenido busca algunas conclusiones o extrae inferencias -explicaciones“contenidas" explícita o implícitamente en el propio texto. La inferencias representan una ventana hacia los procesos cognitivos, actualmente se consideran "el núcleo de la 
OBJETIVOS DE LAS PRÁCTICAS PROFESIONALES DE LA ESCUELA DE

BIBLIOTECOLOGÍA
Tania P. Vera, Roselin R. Esquivia, Yohaliseh C. Muñoz ( pp 29.-56)

comprensión e interpretación de la realidad y, por tanto, uno de los pilares de la cognición humana" (Escudero 2004). En este sentido, del análisis de contenido realizado, se derivan las siguientes inferencias:

$\checkmark \quad$ Los objetivos de las PPII Automatización contemplan la mayor cantidad de elementos. Sin embargo, no se considera la importancia de desarrollar proyectos dentro del área de ciencias de la información.

$\checkmark \quad$ Los objetivos de la PPII: Procesos técnicos en archivos no mencionan el manejo de TIC para el diseño y adecuación del funcionamiento técnico documental, a pesar de que, para la fecha de su redacción ya era un hecho la incorporación de herramientas tecnológicas de información y comunicación en la ejecución de los procesos documentales.

Tampoco se introdujo el componente ético, el cual es fundamental al tratarse estudiantes que van a manejar documentos específicos de empresas e instituciones. No obstante, es el único programa cuyos objetivos incluyen lo concerniente a la conservación del patrimonio documental histórico y cultural

$\checkmark \quad$ En ninguno de los programas de PPII vigentes hasta el 2011 se impulsa explícitamente la actuación del estudiante como agente de cambio en las unidades de información donde se inserta para realizar sus prácticas, considerando que debe ser una de las cualidades de todo egresado: cambiar y transformar, para mejor, las situaciones presentes en los escenarios laborales en los que le toque intervenir profesionalmente.

$\checkmark \quad$ En los objetivos de los programas de PPII tampoco no se estipula con claridad el deber que tiene el profesional para determinar necesidades externas e internas de información en las organizaciones, relativas a funciones, actividades y procesos administrativos.

De la correlación entre el diseño curricular que entró en vigencia desde el 2012 y los sinópticos de los programas correspondientes a las PPII, se obtuvieron los siguientes resultados; con respecto a la frecuencia (F) de aparición de las categorías en los objetivos analizados:

1. 2Ca Actitudinales: Valora su importancia como analista de información para promover la socialización del conocimiento (8) 
OBJETIVOS DE LAS PRÁCTICAS PROFESIONALES DE LA ESCUELA DE BIBLIOTECOLOGÍA $\quad$ Y ARCHIVOLOGÍA
Tania P. Vera, Roselin R. Esquivia, Yohaliseh C. Muñoz ( pp 29.-56)

2. 2Ba Procedimentales: Ejecuta la organización y representación de la información y el conocimiento en distintos soportes y en diversos tipos de unidades, sistemas y servicios de información, considerando estándares (7)

3. $2 \mathrm{Bb}$ Procedimentales: Diseña herramientas para la organización y representación de la información y el conocimiento (5)

4. 2Aa Cognitiva: Analiza los modelos teóricos para la organización y representación de la información y el conocimiento (4)

2Ac Cognitivas: Distingue los diferentes niveles de procesamiento de la información (4)

2Bc Procedimentales: Distingue los diferentes niveles de procesamiento de la información y último

2Cb Actitudinales: Asume una posición crítica en relación con la importancia de la organización y representación de la información y el conocimiento para la maximización de su acceso (4)

Este resultado revela que la competencia más considerada es la actitudinal, se impulsa a los estudiantes a valorar su importancia como analistas de información para promover la socialización del conocimiento. Y de igual modo, la competencia genérica procedimental orientada a la ejecución de la organización y representación de la información y el conocimiento en distintos soportes y en diversos tipos de unidades, sistemas y servicios de información, considerando estándares.

Las categorías con presencia simultánea en los sinópticos analizados son:

2Ba Procedimentales: Asume una posición crítica en relación con la importancia de la organización y representación de la información y el conocimiento para la maximización de su acceso

2Ca Actitudinales: Valora su importancia como analista de información para promover la socialización del conocimiento.

\section{Derivación de inferencias del segundo grupo de documentos}

$\checkmark \quad$ En cuanto a la PPII Teleinformática se observa la omisión de la importancia 
OBJETIVOS DE LAS PRÁCTICAS PROFESIONALES DE LA ESCUELA DE BIBLIOTECOLOGÍA $\quad$ Y ARCHIVOLOGÍA
Tania P. Vera, Roselin R. Esquivia, Yohaliseh C. Muñoz ( pp 29.-56)

de la comunicación, tampoco se vincula el uso de las tecnologías con el intercambio de información, ni se toca los aspectos ambiental y ético

$\checkmark \quad$ Ninguno de los objetivos de la PPII Análisis de la información I incluye la oportunidad que tiene el estudiante de participar activa y solidariamente en el diseño y ejecución de proyectos pertinentes para el desarrollo de la comunidad con ética y responsabilidad social. Tampoco se menciona la importancia del pensamiento crítico para tomar decisiones, detectar problemas y proponer soluciones.

$\checkmark \quad$ Los objetivos que persigue la PPII Análisis de la información II omiten el desarrollo de los procesos de investigación necesarios para el manejo de hechos, ideas, significados y fenómenos con una actitud transformadora, crítica y reflexiva. Y además el importante rol que ejercen las TIC en materia de gestión de contenidos; igualmente, deja de lado lo actitudinal.

\section{CONCLUSIONES Y RECOMENDACIONES}

Algunos objetivos incluidos en los programas de las PPII correspondientes al diseño curricular 1995 establecen logros de aprendizaje que escapan de lo contemplado en el perfil de egreso de ese momento, es posible que esto se deba al extralimitado margen de tiempo que dicho diseño permaneció en vigencia, y lo llevó a estar un tanto desfasado de las nuevas exigencias académicas y profesionales del entorno.

Por otra parte, aspectos de elevada importancia como el trabajo en equipo, la adquisición y desarrollo de competencias para establecer alianzas estratégicas con diversos organismos públicos y privados y con otros servicios de información, en aras de compartir recursos, identificar mejores prácticas y proporcionar un ganar-ganar, no se contempla dentro de los objetivos analizados.

En la mayoría de los objetivos que orientan la ejecución de las PPII se omite lo relacionado con la ética, lo cual pudiera incidir negativamente en los futuros profesionales de la información.

En general, se recomienda promover el pensamiento crítico ante los problemas sociales, lo cual es fundamental para enfrentar los retos laborales con profesionalismo. De igual modo, impulsar la ética como el conjunto de principios y de normas morales que 
OBJETIVOS DE LAS PRÁCTICAS PROFESIONALES DE LA ESCUELA DE BIBLIOTECOLOGÍA $\quad$ Y ARCHIVOLOGÍA
Tania P. Vera, Roselin R. Esquivia, Yohaliseh C. Muñoz ( pp 29.-56)

regulan las actividades humanas; así mismo, el uso de las tecnologías de la información y la comunicación según las demandas de cada contexto y para responder a las tendencias mundiales de desarrollo tecnológico, científico y cultural.

Otro de los aspectos fundamentales que el estudiante debe adquirir es la capacidad para trabajar en equipo. El trabajo en equipo puede hacer que las actividades fluyan de manera más rápida y eficiente.

Se recomienda que los docentes que dictan las PPII evalúen los objetivos contemplados en los programas de cada práctica para efectuar los ajustes necesarios, y contribuir así con el mejoramiento progresivo en la ejecución de las prácticas profesionales II.

\section{REFERENCIAS}

Abela, J. (2000). Las técnicas de Análisis de Contenido: Una revisión actualizada. Granada: Fundación Centro Estudios Andaluces. Universidad de Granada.

Ajá, J. y Albaladejo, C. (2000). La evaluación educativa. Enciclopedia general de la educación. Barcelona: Editorial Océano.

Bartolomé, M. (1981). Estudios y experiencias sobre educación en valores. Madrid: Arcea.

Berelson, B. (1952). Content Analysis in Comunication Research. Glencoe: Free Press.

Berger, C. y Kam, R. (1996). Definitions of Instructional Design. Adapted from "Training and Instructional Design". Applied Research Laboratory, Penn State University. Disponible en: http://www.umich.edu/ ed626/define.html. [Consulta: 2012, Julio 21].

Bligh, D. (2000). "What's the Use of Lectures?". En Gibbs (Ed). Teaching in Higher Education: Theory and Evidence. San Francisco: Jossey-Bass Publishers.

Bracho, M. y Caldera, E. (2002). Programa PPII: Procesos Técnicos en Bibliotecas. Escuela de Bibliotecología y Archivología Militza Bracho y Edixon Caldera. Maracaibo, Venezuela.

Bracho, M. y Peña, T. (2005). Unidad Curricular: PPIII (Pasantías). Escuela de Bibliotecología y Archivología. La Universidad del Zulia. Maracaibo, Venezuela.

Canquíz, L. e Inciarte, A. (2007). Formación integral y competencias profesionales. Ponencia presentada en la VII Reunión Nacional de Currículo y el I Congreso Internacional de Calidad de Innovación en Educación Superior. Caracas del 9-13 de abril.

Disponible

en: 
OBJETIVOS DE LAS PRÁCTICAS PROFESIONALES DE LA ESCUELA DE BIBLIOTECOLOGÍA $\quad$ Y ARCHIVOLOGÍA
Tania P. Vera, Roselin R. Esquivia, Yohaliseh C. Muñoz ( pp 29.-56)

http://portal2.mppeuct.gob.ve/web/uploads/documentos/documentosVarios/pdf10-082010_09:44:33.pdf. [Consulta: 2013, Septiembre 22].

Casarini, M. (2001). Teoría y diseño curricular. (2da. Ed.). México: Editorial Trillas.

Castellano, A. (2008). Desarrollo de las prácticas profesionales, para una integración formativa. Guadalajara: Universidad de Guadalajara.

Chávez, N. (2007). Introducción a Investigación Educativa. Maracaibo: Gráfica González.

Coon, D. (2005). Fundamentos de psicología. (10ª Ed.). México DF:Editorial Thomson.

Cruz, F. (2001). Hábitos de estudio, actitudes y autoestima relacionados con rendimiento académico en estudiantes de enfermería. Revista de EUMEDNET. 3 (23), Universidad Veracruzana, México. [Artículo en línea]. Disponible en: http://www.eumed.net/rev/ced/23/fcn.htm. [Consulta: 2013, Febrero 01].

Departamento tecnología y práctica educacional (1997). Programa de la asignatura práctica profesional para la docencia nivel II. Universidad del Zulia. Maracaibo, Venezuela.

Escudero, I. (2004). Procesamiento de inferencias elaborativas en la comprensión del discurso y según el tipo de texto. Tesis de doctorado no publicada. Universidad Autónoma de Madrid, Madrid.

Escuela de Bibliotecología y Archivología. (1995). Diseño Curricular de la Escuela de Bibliotecología y Archivología. Universidad del Zulia. Maracaibo, Venezuela.

Fernández, O.; Battigelli, C.; Luque, R.; Gómez, C.; Finol, W.; Pérez, E. et al. (2002). Programa práctica profesional para la docencia Nivel I. Departamento de tecnología y práctica educacional. LUZ. Maracaibo, Venezuela.

Ferrer, L. (s/a). Programa PPII: Análisis de la Información. Universidad del Zulia. Escuela de Bibliotecología y Archivología. Maracaibo, Venezuela.

Ferrer, L. (2010). Programa PPII: Análisis de la Información. Universidad del Zulia. Escuela de Bibliotecología y Archivología. Maracaibo, Venezuela 